\section{Superconducting Thin Films}

WE wish to report some preliminary results on the superconductive properties of lead and tin films between $5 \times 10^{-7} \mathrm{~cm}$. and $2 \times 10^{-5} \mathrm{~cm}$. thick, obtained by condensation from the vapour. The films were deposited on a glass surface at $4 \cdot 2^{\circ} \mathrm{K}$. which carried four platinum electrodes, the conductivity measurements being made by means of a compensation apparatus; the film thicknesses were determined from the difference in mass of the source before and after evaporation. In order to ensure the

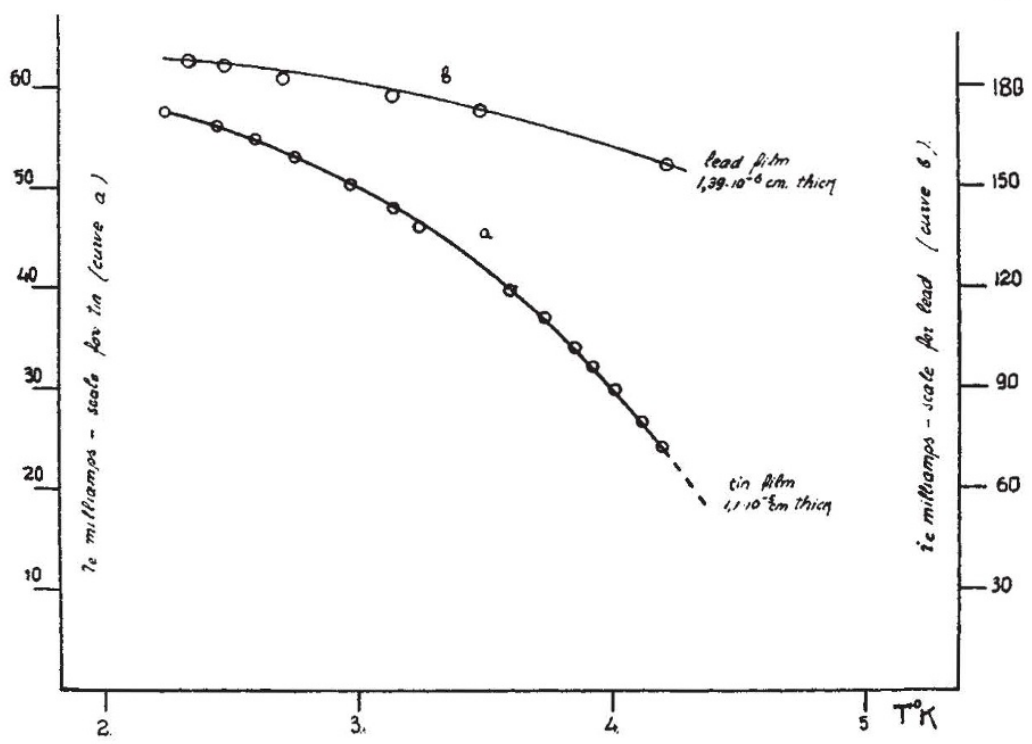

$1^{\circ}$ higher than for tin in bulk. For lead this extrapolation is not possible, owing to the flat course of the $i_{c}-T$ curve (Curve $b$ ), but the curve suggests that here also the transition temperature may be higher than for lead in bulk $\left(7 \cdot 2^{\circ} \mathrm{K}\right.$.).

A significant feature of the results is that the superconductive properties underwent a marked change if, after deposition, the films were warmed to room temperature (probably causing recrystallization), and then recooled. Thus in the case of tin the transition temperature dropped to about $3 \cdot 7^{\circ} \mathrm{K}$. (the value for bulk tin); for a temperature equally far below the transition temperature the magnetic fields required to restore resistance became rather smaller, and $i_{c}$ became rather greater.

Since all the properties (except the sharpness of the transition between super- and normal conductivity) are very reminiscent of those of super-conductive alloys, it seemed that the anomalously high transition temperature of freshly deposited tin might be connected with the presence of impurities; variation of cleanliness of the condensation conditions, however, caused no change of the high transition temperature, so it is likely that this is genuinely characteristic of the structure of films deposited at $4 \cdot 2^{\circ} \mathrm{K}$. How far the other anomalous properties are connected with the structure and how far with the thinness of the

cleanest possible conditions, the condensation and measurements were made in a sealed-off high-vacuum apparatus.

Even the thinnest films investigated showed superconductivity. The resistance was restored in a single abrupt jump if the measuring current was increased beyond a critical value $i_{c}$ characteristic of the particular film and temperature; further increase of current produced no appreciable change of resistance. On again reducing the current, the resistance disappeared in a single jump, but at a lower value of current than $i_{c}$; the whole resistance-current curve could be reproduced any number of times. The value of $i_{c}$ at a given temperature decreased with the thickness of the film, and was in general of the order of magnitude of some milliamperes; in all cases this was very much smaller than could be expected from Silsbee's hypothesis, supposing the critical field to be the same as for the bulk metal.

Actually, however, the magnetic fields required to restore the resistance were not lower, but much higher than for the bulk metal; thus a magnetic field even as high as several thousand gauss, applied parallel to the current, reduces the value of the critical current, but this influence is only slight (especially for the thinner films). With increase of the film thickness the magnetic field required to destroy superconductivity decreases.

The critical current decreases with increase of temperature, and in the case of tin, it was possible to estimate the transition temperature by extrapolation of the accompanying $i_{c}-T$ curve (Curve $a$ ) to zero $i_{c}$. This suggested a transition temperature of about $4 \cdot 7^{\circ} \mathrm{K}$. for all the tin films, which is about films, must remain a question for further investiga-
tion, but the results, so far, suggest that the structure can scarcely be responsible alone.

\section{A. Shalnikov.}

Institute for Physical Problems, Academy of Sciences of the U.S.S.R., Moscow. April 27.

\section{Melting and Structure of Long-Chain Ketones}

A NUMBER of isomeric long-chain ketones have been prepared and specially purified, in the course of investigations on the solid-liquid transition in longchain compounds ${ }^{1}$. As will be clear from the diagram for the ketones $\left.\mathrm{C}_{n} \mathrm{H}_{2 n+1} \mathrm{COC}_{(16-n)}\right) \mathrm{H}_{33-2 n}$, the setting points depend in a remarkable way on the position of the CO dipole in the long-chain molecule. The maximum difference (between $\mathrm{C}_{8} \mathrm{H}_{17} \mathrm{COC}_{8} \mathrm{H}_{17}$ and $\mathrm{C}_{5} \mathrm{H}_{12} \mathrm{COC}_{11} \mathrm{H}_{23}$ ) of $9 \cdot 2^{\circ}$ may be compared with the difference in setting points between $\mathrm{C}_{5} \mathrm{H}_{11} \mathrm{COC}_{12} \mathrm{H}_{23}$ (s.p. $40 \cdot 8^{\circ}$ ) and the corresponding paraffin $\mathrm{C}_{17} \mathrm{H}_{36}$ (s.p. $21 \cdot 6^{\circ}$ ).

In order to verify that this behaviour is not confined to chains with an odd number of carbon atoms, ketones of the series $\left.\mathrm{C}_{n} \mathrm{H}_{2 n+1} \mathrm{COC}_{(17-n)}\right) \mathrm{H}_{35-2 n}$ have also been prepared, and show a corresponding behaviour. A survey of published melting points of other ketones suggests that this effect is quite general.

In view of the comparatively simple crystal structure of long-chain compounds ${ }^{2,3}$, these observamodels of the melting of erystal lattices. A detailed analysis of the crystal structure of a long-chain tions are of interest for theories and mechanical 